\title{
Second coming: the re-emergence and modernization of immunotherapy by vaccines as a component of leprosy control
}

\author{
Malcolm S Duthie ${ }^{*, 1}$, Corey Casper ${ }^{1} \&$ Steven G Reed ${ }^{1}$ \\ ${ }^{1}$ From the Infectious Disease Research Institute, 1616 Eastlake Ave E, Suite 400, Seattle, WA 98102, USA \\ *Author for correspondence: Tel.: +206 381 0883; Fax: +206 381 3678; mduthie@idri.org
}
“LepVax is a defined subunit vaccine incorporating a recombinant fusion protein with a synthetic TLR4 agonist in a stable emulsion formulation."

First draft submitted: 19 June 2018; Accepted for publication: 28 June 2018; Published online:

12 October 2018

Keywords: adjuvant $\bullet$ attenuated $\bullet$ inflammatory $\bullet$ leprosy $\bullet$ protein

Leprosy (Hansen's disease) is a complex peripheral neurological disorder caused by Mycobacterium leprae infection. It is among the leading causes of nontraumatic peripheral neuropathies worldwide. The past three decades have witnessed some impressive advances in leprosy control, with the reduction of new leprosy cases largely attributed to widespread dissemination and use of multidrug therapy (MDT) through the generous donation of both drugs and logistical support from organizations such as Novartis Foundation and World Health Organization [1]. Leprosy remains a critical public health priority, however, and it was targeted by the London Declaration on Neglected Tropical Diseases for elimination by 2020. Although incident cases of leprosy have been reduced from the levels observed in the 1980s, these reductions have stalled over the last decade and over 200,000 new patients are still reported as developing leprosy worldwide each year [2-4]. The considerable proportion of new cases that emerge among children indicates that the transmission of $M$. leprae is ongoing, and an increased proportion of cases with disabilities suggests that proper diagnosis is being delayed. It is also widely believed that officially reported case numbers are a gross underestimate of the actual number of leprosy cases/ $M$. leprae-infected individuals [3,5]. New intervention strategies are required to maintain and promote further reductions. In 2016, WHO launched the 'Global Leprosy Strategy 2016-2020: accelerating toward a leprosy-free world', which aims to reinvigorate efforts to control leprosy and avert disabilities, especially among children [2].

While prophylactic rifampicin treatment is currently being evaluated as a means for early drug treatment of subclinical $M$. leprae infection and interruption of transmission in a limited number of settings, similar previous trials have shown chemoprophylaxis to have only a transient impact on local incidence (typically 2-3 years) [6-8]. This is likely due to drug efficacy being restricted to already infected individuals and protection only being afforded while the drug is being taken (i.e., waning with incomplete adherence or once use of the drug has terminated). Sustained elimination likely requires robust protection in both infected people and individuals at elevated risk of future $M$. leprae infection. An effective vaccine would have the potential to address the limitations of drug therapy but the evaluation of vaccines for the prevention of leprosy is complicated by multiple factors. Among these, the relatively low attack rate in the general population and the long incubation period (estimated to be an average of 7-10 years) mandates prolonged monitoring of a large number of people. Focusing interventions to individuals at the highest risk (contacts of highly infected patients), many of whom may already be infected with $M$. leprae, presents a more tenable strategy with which to observe disease emergence in a shorter timeframe and lower number of trial enrollees. Adding a vaccine to standard care (MDT) to determine if this improves the clinical outcome of well-characterized leprosy patients (i.e., people known to be infected with M. leprae), however, provides the most efficient evaluation of both safety and efficacy. Success as a chemo-, immuno-therapeutic intervention at the postexposure level would augur well for transition from therapeutic use to preventative administration in a broader population. 
Several killed or attenuated vaccines were evaluated for their protective efficacy against $M$. leprae but research efforts waned with the relative success of MDT [6]. Besides the continued use of BCG, which confers only partial protection [9-11], two vaccines have recently been positioned for use in clinical trials with the goal of providing long-term protection and sustained leprosy control. The first of these is Mycobacterium indicus pranii (heat-killed MIP; previously known as Mycobacterium w). MIP was initially developed at the National Institute of Immunology, India as an adjuvant component for use in vaccines and has been evaluated in clinical trials for tuberculosis and various tumors [12,13]. MIP is now licensed and produced as Immuvac/Cadi- 05 by Cadila Pharmaceuticals for use as an adjunctive therapy of leprosy [14]. The addition to MDT of heat-killed MIP vaccine as an immunomodulator leads to more rapid attainment of smear negativity in leprosy patients relative to the average fall in BI with standard MDT [15]. Although live attenuated or killed mycobacteria vaccines have generally been regarded as being well tolerated in general populations, (reimmunization of close contacts of leprosy patients with BCG has been common practice in some countries [11]) some fear that immunization to boost inflammatory T-cell responses against $M$. leprae will precipitate disease or induce inflammatory reversal reactions. Indeed, an important previously anecdotal observation that is now being documented is the precipitation of paucibacillary leprosy following BCG immunization of individuals believed to harbor asymptomatic $M$. leprae infection $[16,17]$. In a recently reported double-blind trial, eight reactional episodes were observed during the initial 6 months of therapy in the intervention group (five reversal reactions, three erythema nodosum leprosum from a total of 46 patients), compared with four in the control group (three reversal reactions, one erythema nodosum leprosum from 44 patients) [18]. It was mentioned, but not documented, that at 6-12 months and after 12 months, the incidence of reactions was lower in the MIP intervention group. In 2017, an expert committee of the Indian Council of Medical Research and the Directorate of Health Services determined to treat leprosy patients in five districts of high endemicity with autoclaved MIP in conjunction with MDT. It was also recommended that immediate contacts of the patients will be immunized with two doses of MIP, provided with a 6-month interval between inoculations [19].

The second vaccine to advance to clinical application is LepVax, a vaccine we have developed specifically for leprosy [20]. LepVax is a defined subunit vaccine incorporating a recombinant fusion protein with a synthetic TLR4 agonist in a stable emulsion formulation. The antigenic component of LepVax is LEP-F1, expressed by E. coli as an $89 \mathrm{kDa}$ chimeric recombinant fusion protein consisting of the tandem linkage of four $M$. leprae antigens ( $\mathrm{m} / 2531$, $m l 2380, m l 2055$ and $m l 2028$ ). These antigens were selected on the basis of immune recognition by paucibacillary leprosy patients and $M$. leprae-exposed individuals. When evaluated in the mouse footpad system as pre-exposure prophylaxis, LepVax produced an antigen-specific Th1 response and significantly reduced the infectious burden. When evaluated in nine-banded armadillos (a symptomatic disease model) as postexposure prophylaxis, provision of LepVax three-times, at monthly intervals significantly delayed motor nerve injury relative to that observed in control animals. Importantly, administration of a similarly adjuvanted subunit TB vaccine (ID93 + GLA-SE) did not delay or alleviate nerve function deficits, indicating that efficacy was likely not mediated by adjuvantlike activity but rather in an antigen-specific manner. Also important is that the beneficial outcome provided by LepVax directly contradicted the rapid and severe precipitation to abnormal nerve conduction that occurred in armadillos that received postexposure BCG immunization. Data from two independent evaluations demonstrated that postexposure prophylaxis with LepVax was safe, and alleviated and delayed the neurologic damage caused by $M$. leprae infection. More recently a Phase I safety evaluation in healthy adults in the USA was initiated and follow-up will be completed soon (ClinicalTrials. gov Identifier: NCT03302897).

In summary, while leprosy currently appears to be under some form of containment, its elimination as a global health concern appears to be just beyond reach. We believe that the emergence of MIP and LepVax provides important building blocks toward disease elimination. A combined chemo- and immunotherapeutic approach in a post-exposure setting could provide immediate impact by preventing disease and transmission. In light of positive trial data, the next steps entail carrying out assessments of public health impact, perception and acceptability of vaccination strategies and economic analyses. These assessments are required to facilitate the integration of vaccinebased strategies into pre-exposure settings that can modernize leprosy programs and provide sustained control. Research evaluating the safety and utility of these promising leprosy vaccine candidates in the prevention of leprosy and its complications should be considered a critical public health priority.

\section{Author contributions}

Each author made substantial, direct and intellectual contributions to this submission. 


\section{Acknowledgements}

We thank P Saunderson, T Gillis, W Britton, P Brennan and A Ginsberg for discussion and comments as members of the Scientific Steering Committee during the development of LepVax, as well as each of our collaborative partners in leprosy-endemic countries for their invaluable contributions. This program has benefited from reagents and services available through NIAID/HRSA Interagency Agreement number AAl15006-004-00000, National Hansen's Disease Programs. Our deepest gratitude to the leprosy patients, contacts and control individuals who provided samples without which our program would not have advanced.

\section{Financial \& competing interests disclosure}

Leprosy research at IDRI has been funded by grants from American Leprosy Missions (including contributions from DAHW [German Leprosy and Tuberculosis Relief Association], Damien Foundation Belgium, effect:hope [The Leprosy Mission Canada], FAIRMED, Fondation Raoul Follereau, Leprosy Relief Canada and Netherlands Leprosy Relief), Order of Malta (MaltaLep), The Heiser Program for Research in Leprosy and Tuberculosis of The New York Community Trust. SG Reed and MS Duthie have a patent pending on the development of defined subunit vaccines for leprosy. The authors have no other relevant affiliations or financial involvement with any organization or entity with a financial interest in or financial conflict with the subject matter or materials discussed in the manuscript apart from those disclosed.

No writing assistance was utilized in the production of this manuscript.

\section{References}

1. World Health Organisation. Global leprosy update, 2016: accelerating reduction of disease burden. Wkly Epidemiol. Rec. 92(35), 501-519 (2017).

2. Rao PN. Global leprosy strategy 2016-2020: issues and concerns. Indian J. Dermatol. Venereol. Leprol. 83(1), 4-6 (2017).

3. Smith WC, van Brakel W, Gillis T, Saunderson P, Richardus JH. The missing millions: a threat to the elimination of leprosy. PLoS Negl. Trop. Dis., 9(4), e0003658. (2015).

4. Hotez PJ. Ten failings in global neglected tropical diseases control. PLoS Negl. Trop. Dis. 11(12), 0005896 (2017).

5. Salgado CG, Barreto JG, da Silva MB et al. Are leprosy case numbers reliable? Lancet Infect. Dis. 18(2), 135-137 (2018).

6. Duthie MS, Balagon MF. Combination chemoprophylaxis and immunoprophylaxis in reducing the incidence of leprosy. Risk Manag. Healthc. Policy 9, 43-53 (2016).

7. Barth-Jaeggi T, Steinmann P, Mieras L et al. Leprosy post-exposure prophylaxis (LPEP) programme: study protocol for evaluating the feasibility and impact on case detection rates of contact tracing and single dose rifampicin. BMJ Open 6(11), e013633 (2016).

8. Tiwari A, Mieras L, Dhakal K et al. Introducing leprosy post-exposure prophylaxis into the health systems of India, Nepal and Indonesia: a case study. BMC Health Serv. Res. 17(1), 684 (2017).

9. Setia MS, Steinmaus C, Ho CS, Rutherford GW. The role of BCG in prevention of leprosy: a meta-analysis. Lancet Infect. Dis. 6(3), 162-170 (2006).

10. Duppre NC, Camacho LA, da Cunha SS et al. Effectiveness of BCG vaccination among leprosy contacts: a cohort study. Trans. R. Soc. Trop. Med. Hyg. 102(7), 631-638 (2008).

11. Cunha SS, Alexander N, Barreto ML et al. BCG revaccination does not protect against leprosy in the Brazilian Amazon: a cluster randomized trial. PLoS Negl. Trop. Dis. 2(2), e167 (2008).

12. Sharma SK, Katoch K, Sarin R et al. Efficacy and safety of Mycobacterium indicus pranii as an adjunct therapy in category II pulmonary tuberculosis in a randomized trial. Sci. Rep. 7(1), 3354 (2017).

13. Talwar GP, Gupta JC, Mustafa AS et al. Development of a potent invigorator of immune responses endowed with both preventive and therapeutic properties. Biologics 11, 55-63 (2017).

14. Talwar GP, Singh P, Atrey N, Gupta JC. Making of a highly useful multipurpose vaccine. J. Transl. Med. 1(3), 69-73 (2016).

15. Kamal R, Natrajan M, Katoch K, Arora M. Clinical and histopathological evaluation of the effect of addition of immunotherapy with Mw vaccine to standard chemotherapy in borderline leprosy. Indian J. Lepr. 84(4), 287-306 (2012).

16. Duppre NC, Camacho LA, Sales AM et al. Impact of PGL-I seropositivity on the protective effect of BCG vaccination among leprosy contacts: a cohort study. PLoS Negl. Trop. Dis. 6(6), e1711 (2012).

17. Richardus RA, Butlin CR, Alam K, Kundu K, Geluk A, Richardus JH. Clinical manifestations of leprosy after BCG vaccination: an observational study in Bangladesh. Vaccine 33(13), 1562-1567 (2015).

18. Kamal R, Pathak V, Kumari A, Natrajan M, Katoch K, Kar HK. Addition of Mycobacterium indicus pranii vaccine as an immunotherapeutic to standard chemotherapy in borderline leprosy: a double-blind study to assess clinical improvement (preliminary report). Br. J. Dermatol. 176(5), 1388-1389 (2017).

19. Talwar GP, Gupta JG. Launching of immunization with the vaccine Mycobacterium indicus pranii for eradication of leprosy in India. Int. J. Vaccine Res. 2(3), 1-5 (2017).

20. Duthie MS, Pena MT, Ebenezer GJ et al. LepVax, a defined subunit vaccine that provides effective pre-exposure and post-exposure prophylaxis of M. leprae infection. NPJ Vaccines 3, 12 (2018). 
Editorial Duthie, Casper \& Reed 